\title{
Guideline Development Summary
}

\author{
Werner J. Becker on behalf of the Canadian Headache Society Acute Migraine \\ Treatment Guideline Development Group
}

Can J Neurol Sci. 2013; 40: Suppl. 3 - S73-S76

The development of this guideline for the acute pharmacological treatment of migraine is described in this appendix, using the 23 items of the AGREE instrument for the appraisal of practice guidelines as a framework. ${ }^{1}$

\section{Objectives:}

To assist the practitioner to:

a. Choose an appropriate acute medication for an individual with migraine, based on current evidence in the medical literature.

b. Use the chosen medication in the most effective manner.

c. Reduce the headache-related disability suffered by individuals with migraine.

d. Use the best evidence in the medical literature in the clinical context of overall migraine treatment.

\section{The clinical question addressed:}

a) Which acute medication should be prescribed for an individual patient in a specific clinical situation?

\section{The target population:}

a) Adults with episodic migraine (patients who experience migraine headache attacks on less than 15 days/month). It does not include recommendations for pediatric patients and for the emergency room management of acute migraine. Although this guideline may have relevance to patients with chronic migraine (headache on 15 days a month or more, with diagnostic criteria for migraine met on at least eight days a month), many of the clinical trials reviewed for this guideline did not include patients with headache frequencies of this magnitude.

\section{Professional groups involved in the creation of these guidelines:}

a) These guidelines were produced by the Canadian Headache Society. Health professionals involved in development of the guideline included neurologists, pharmacists, family physicians, and nurses with a special interest in headache.

\section{Patient views and preferences:}

a) Patient expectations, views and preferences were obtained from the medical literature. A published patient survey indicated that an overwhelming majority of patients consider complete relief of head pain, no recurrence, and rapid onset of action as important or very important attributes of acute migraine therapy. ${ }^{2}$ Generally, patients want a drug that provides complete headache relief. ${ }^{3}$
These patient values were considered in the production of this guideline, and many clinical trials use endpoints which made this possible.

b) Patient views and experiences were also obtained at the Canadian Migraine Forum ${ }^{4-6}$, which was hosted by the Canadian Headache Society prior to the development of these guidelines.

\section{The target users for this guideline:}

a) This guideline is intended primarily for physicians who treat patients with migraine, including both family physicians, and specialists. Other health professionals who treat patients with migraine may also find this guideline helpful.

b) Although they are not the primary target users of this guideline, this guideline may also be helpful to patients with migraine and their families.

\section{Pre-testing of the guidelines:}

These guidelines have not been specifically pre-tested among the intended end users. They have however been created by experienced clinicians with extensive experience in medication use for migraine. This experience has been utilized to make the guidelines as clear and practical as possible.

\section{Systematic methods were used to search for evidence with regard to the individual drugs assessed:}

The recommendations for individual drugs in the Guideline are based on a targeted review (Section 2 of the Guideline). Primarily meta-analyses and systematic reviews were included. Where these were not available for a drug or were out of date, individual clinical trial reports were utilized. Only double-blind randomized clinical trials with placebo or active drug controls were included in the analysis for the targeted review provided in Section 2. For other aspects of the guideline document which deal with more general questions pertinent to acute migraine treatment where randomized trials do not exist, a general literature review was done and expert opinion was used to draw conclusions regarding suggested management. These conclusions are clearly labelled as "Expert consensus" rather than "Recommendations" in order to avoid confusion.

\footnotetext{
From the University of Calgary and the Hotchkiss Brain Institute, Calgary, Alberta, Canada.

Received June 9, 2013. Final Revisions Submitted June 22, 2013. Correspondence to: W.J. Becker, Division of Neurology, 12th Floor, Foothills Hospital, 1403 29th St NW, Calgary, Alberta, T2N 2T9, Canada.
} 
For the targeted review, a detailed search strategy was employed to find relevant published clinical trials of drugs used in Canada for the acute treatment of migraine in adults.

a) A MEDLINE search of the English language literature for analgesics, NSAIDs, ergot derivatives, and triptans was performed. Only randomized, controlled trials (RCTs) and meta-analyses/systematic reviews of acute migraine medications in adults were included. The initial search was limited to the years 1996 - May 2006 (first Canadian migraine guidelines were published in 1997). The search was updated in May 2010, and again in May 2012.

b) The Cochrane Collaboration $\AA$ and EMBASE were also searched for systematic reviews/meta-analyses.

c) Search terms used were:

i. exp. migraine disorders, and

ii. sumatriptan or almotriptan or eletriptan or naratriptan or rizatriptan or zolmitriptan or frovatriptan or "triptan", or

iii. exp. anti-inflammatory agents, non-steroidal, or

iv. exp. aspirin, or acetaminophen, or exp. analgesics, or

v. ergotamine or dihydroergotamine, or

vi. exp. barbiturates or butalbital, or

vii. metoclopramide or domperidone or dimenhydrinate or exp. antiemetics

viii. limits: human, adults, English, randomized controlled trial (RCT) or meta-analysis

\section{Criteria used for including / excluding evidence identified} by the search:

a) Studies were required to be prospective, randomized, double blind, controlled trials of drugs used for acute migraine treatment.

b) Trials comparing treatments to placebo or an active comparator were included.

c) Both parallel group and cross-over designs were acceptable.

d) Study participants had to be adults and meet $\mathrm{IHS}^{7}$ or Ad Hoc Committee on Classification of Headache (JAMA. $1962 ; 179: 717-8)$ criteria for the diagnosis of migraine headache, or provide sufficient detail of the headache characteristics to support the diagnosis of migraine (for studies conducted prior to development of Ad Hoc criteria).

e) The literature search was limited to agents commonly used in clinical practice, as explained in the text.

f) Trials of patients with chronic daily headache (headache on $\geq 15$ days per month), chronic tension type headache or transformed migraine were not included.

\section{Methods used to formulate the recommendations:}

a) Abstracts of studies and meta-analyses identified by the literature search were screened for eligibility by two independent reviewers. Papers that could not be excluded with certainty from this process were reviewed in full. Papers passing the initial screening process were retrieved and the full text was reviewed.

b) Recommendations were graded based on the principles of the Grading of Recommendations Assessment, Development and Evaluation (GRADE) Working Group. ${ }^{8,9}$ c) Papers were reviewed independently by two reviewers and graded with regard to methodological quality. The literature review, and draft recommendations were presented by IW, and WJB to a group of six experts from the Canadian Headache Society on Oct 29, 2011 in Montreal, and consensus reached through discussion and mutual agreement. The recommendations were graded according to the Grade criteria, and levels of evidence were assigned to the recommendations.

d) The guidelines were presented by IW and WJB and further discussed and validated with a group of four Canadian headache experts on June 7, 2012 in Ottawa, in conjunction with the Canadian Neurological Sciences Federation.

e) The draft guideline was then extensively circulated by email to the entire guideline development group (including all the authors) and extensive commentary and feedback was obtained from many. The drafts were then updated for external review.

f) The guidelines were sent to two external reviewers who had not been involved in the guideline development to that point, a family physician with a special interest in headache and pain, and a pharmacist with special expertise in pain management. All their suggestions were considered by the guideline authors, and the great majority incorporated into the guideline document.

11. Health benefits, side effects, and risks of the recommendations were considered:

a) The main outcome measures used were pain freedom (pain-free) at two hours and headache relief (pain reduced from severe or moderate to mild or none) at two hours. These outcomes are important to patients, (2) and were also the main outcomes used in many clinical trials. Other outcomes important to patients including headache recurrence rates and adverse events were also considered.

b) The prevalence of adverse events was analyzed in the studies reviewed, and these were considered in the recommendations. The recommendation grading system used (GRADE) considers adverse events in the grading of recommendations.

\section{The link between the recommendations and the evidence on which they are based:}

a) In Section 2 of the guidelines, each drug and the evidence for its efficacy are presented and discussed. All the references on which the recommendation for that drug is based are provided. Tables are provided which summarize the evidence contained in the references.

\section{External review of the guideline:}

a) The guidelines were externally reviewed by two experts not involved in the Guideline Development Group. Reviewers were asked to critically review the guidelines, and their feedback was considered. These reviewers included:

i) A family physician with special expertise in headache and pain

ii) A pharmacist with special expertise in pain 
14. The Guidelines were developed on behalf of the Canadian Headache Society. The executive of the Society has undertaken to review and update the guidelines every three years.

15. Every attempt has been made to provide a concrete and precise description of which management is appropriate in a given clinical situation and in a particular patient group, as permitted by the body of evidence:

a) In addition to the evidence-based targeted review for each acute drug, several sections were added to the guidelines to clarify which management is appropriate in which clinical situation.

b) Section 1 of the guideline includes a section on "General principles of acute migraine therapy" that discusses the available medication formulations (tablets, injections, etc) and the various general treatment approaches which are available. This section also discusses how to choose between treatment options.

c) Section 3 provides a detailed approach on how to choose a medication for a specific patient. The available acute treatments have been organized into treatment strategies, and the specific clinical situations in which each strategy should be considered are outlined in detail.

d) As the evidence for some of the required decision making is limited, it is clearly stated when a general literature review and expert opinion is the basis for a conclusion. To avoid confusion, when non-randomized studies and expert opinion is the basis for a conclusion regarding how patients in specific clinical situations should be managed, the suggested management is labelled "Expert consensus" rather than a recommendation. The term "recommendation" is reserved for recommendations based on evidence from randomized double blind controlled clinical trials.

16. Different possible treatment options for the reduction of migraine frequency in addition to pharmacological prophylaxis are mentioned:

a) The great majority of patients with migraine in Canada utilize an acute medication for treatment of their migraine attacks. ${ }^{10}$ These are not sufficient for some patients, however, and in Section 1, it is clearly stated that for patients with frequent attacks who are at risk of medication overuse headache because they require their acute medication too frequently, behavioural approaches to migraine management and prophylactic medications should be considered in addition to acute medications. This message is reinforced for migraine management in general in Section 3 which deals with treatment strategies in detail.

17. The guideline is structured so that users may find the most relevant recommendations easily:

a) All the recommendations and expert consensus statements are numbered in the document, and printed so that they stand out from the rest of the text.

b) Table 8 , Section 2 provides a list of all the drugs reviewed, the strength of the recommendations for their use, and the level of evidence supporting that recommendation. c) Table 2, Section 3 lists all the acute treatment strategies, and Tables 11a and 11b list all the drugs involved in each treatment strategy.

d) Table 9, Section 3 provides a list of all the drugs recommended, dosages, formulations, their contraindications, and their adverse effects.

e) A list of the various sections and appendices included in this guideline is given under the heading "Guideline structure" in order to assist the reader to access any section of interest easily.

18. Tools have been made available to assist in dissemination and implementation:

a) A summary document for family physicians has been included (Section 4).

b) Tables $11 \mathrm{a}$ and $11 \mathrm{~b}$ in Section 3 summarize the acute migraine treatment strategies, including the medications used in each. They also indicate when each strategy should be used.

c) A guideline summary for patients and the public has been included (Section 5).

d) A patient leaflet which describes acute migraine treatment has been included (Appendix 2).

e) A patient headache diary sheet together with instructions for completion is provided (Appendix 3).

19. Organizational barriers to applying the recommendations of this guideline have been addressed below:

a) Individuals with migraine who currently seek medical attention usually do so through the offices of family physicians and specialists, primarily neurologists. Both these groups are able to prescribe medications, so no significant organizational barriers exist to the prescribing of acute medications as recommended in these guidelines.

b) The prescription of acute migraine medications does require patient follow up for optimal benefit to be achieved. This guideline might reduce the burden of this follow up if physicians are more likely to choose the best drug for the patient first as a result of these guidelines.

c) Individuals with migraine may also seek over-the-counter treatment in pharmacies. Pharmacists are one of the most accessible of health care professionals. This guideline may assist pharmacists in recommending over-the-counter drugs when appropriate, or referring patients to their physician when prescription medications are required or the patient may be suffering from medication overuse headache. This may help reduce the burden of migraine.

20. Potential effects of these guidelines on the need for additional resources:

a) This guideline might potentially increase triptan use as compared to the less expensive codeine containing analgesics which are commonly used or over-used in Canada. It is possible that they might increase the overall costs of drugs used for migraine, but as the triptans are more effective than the combination analgesics for most patients, the indirect costs of migraine, such as missed 
work, should be reduced much more. The indirect costs of migraine (missed work, etc) are much greater than the direct costs (medications, etc). ${ }^{11}$

b) This guideline may also reduce the prevalence of medication overuse headache, a condition which imposes a huge economic and social burden on patients and society. ${ }^{11}$

\section{Options for measuring guideline adherence:}

a) There are a number of options which could be considered to measure guideline adherence in a medical practice. Practice audits could be done to see if patients were treated according to the appropriate treatment strategy. These could also assess what proportion of patients with migraine were receiving acute medications with strong recommendations for use, as opposed to drugs with weak recommendations and drugs not recommended for routine use. Finally, some drugs have strong recommendations against their use. Use of these drugs could also be assessed.

b) Guideline adherence at a regional level could also be evaluated through an analysis of prescriptions for patients with migraine. NSAID and triptan use, for example could be compared with the use of drugs not recommended for routine use such as opiate-containing combination analgesics.

\section{Guideline development and external funding:}

a) This guideline was developed without external funding. All participants volunteered their time. Some minor travel expenses were paid by the Canadian Headache Society.

23. All members of the guideline development group have declared any existing conflict of interest. This has been done in the title page of the guidelines.

\section{REFERENCES}

1. AGREE C. Development and validation of an international appraisal instrument for assessing the quality of clinical practice guidelines: the AGREE project. Qual Saf Health Care. 2003 Feb; 12(1):18-23.

2. Lipton RB, Hamelsky SW, Dayno JM. What do patients with migraine want from acute migraine treatment? Headache. 2002; 42:3-9.

3. Malik SN, Hopkins M, Young WB, Silberstein SD. Acute migraine treatment: patterns of use and satisfaction in a clinical population. Headache. 2006;46:773-80.

4. Becker WJ, Gladstone JP, Aube M. Migraine prevalence, diagnosis, and disability. Can J Neurol Sci. 2007;34:S3-9.

5. Becker WJ, Gawel M, Mackie G, South V, Christie SN. Migraine treatment. Can J Neurol Sci. 2007;34:S10-9.

6. Becker WJ, Giammarco R, Wiebe V. Moving forward to improve migraine management in Canada. Can J Neurol Sci. 2007;34: S20-6.

7. Headache Classification Subcommittee of the International Headache, Society. The International Classification of Headache Disorders: 2nd edition. Cephalalgia. 2004;24:9-160.

8. Guyatt GH, Oxman AD, Vist GE, et al. GRADE: an emerging consensus on rating quality of evidence and strength of recommendations. BMJ. 2008;336:924-6.

9. Guyatt GH, Oxman AD, Kunz R, et al. What is "quality of evidence" and why is it important to clinicians? BMJ. 2008;336:995-8.

10. Cooke LJ, Becker WJ. Migraine prevalence, treatment and impact: the canadian women and migraine study. Can J Neurol Sci. 2010;37:580-7.

11. Linde M, Gustavsson A, Stovner LJ, et al. The cost of headache disorders in Europe: the Eurolight project. Eur J Neurol. 2012; 19:703-11. 